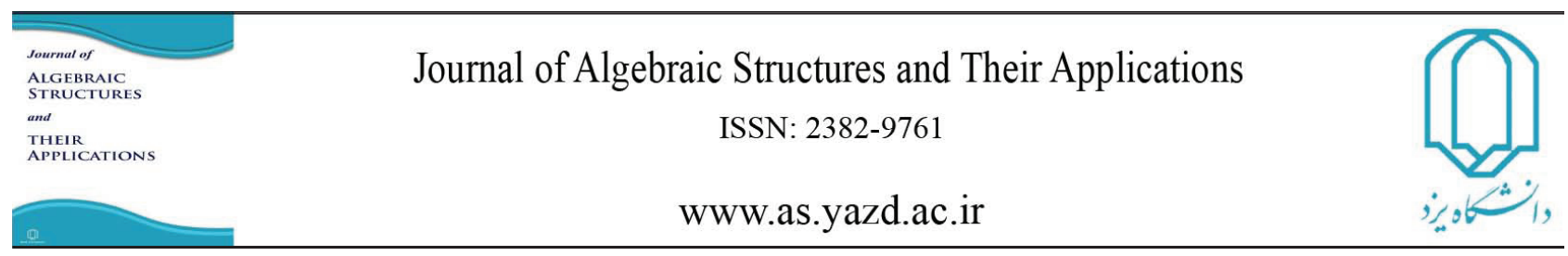

Algebraic Structures and Their Applications Vol. 4 No. 1 ( 2017 ) pp 19-32.

\title{
THE MAIN EIGENVALUES OF THE UNDIRECTED POWER GRAPH OF A GROUP
}

\author{
MEHRNOOSH JAVARSINEH AND GHOLAM HOSSEIN FATH-TABAR*
}

Communicated by A.R. Ashrafi

\begin{abstract}
The undirected power graph of a finite group $G, P(G)$, is a graph with the group elements of $G$ as vertices and two vertices are adjacent if and only if one of them is a power of the other. Let $A$ be an adjacency matrix of $P(G)$. An eigenvalue $\lambda$ of $A$ is a main eigenvalue if the eigenspace $\varepsilon(\lambda)$ has an eigenvector $X$ such that $X^{t} \mathbf{j} \neq 0$, where $\mathbf{j}$ is the all-one vector. In this paper we want to focus on the power graph of the finite cyclic group $\mathbb{Z}_{n}$ and find a condition on $\mathrm{n}$ where $P\left(\mathbb{Z}_{n}\right)$ has exactly one main eigenvalue. Then we calculate the number of main eigenvalues of $P\left(\mathbb{Z}_{n}\right)$ where $n$ has a unique prime decomposition $n=p^{r} p_{2}$. We also formulate a conjecture on the number of the main eigenvalues of $P\left(\mathbb{Z}_{n}\right)$ for an arbitrary positive integer $n$.
\end{abstract}

\section{INTRODUCTION}

All the groups and graphs are assumed to be finite. There are several constructions of graphs from groups like Cayley graphs, Schreier coset graph, orbital digraph and etc. which http://dx.doi.org/10.29252/asta.4.1.19

MSC(2010): Primary:05C50.

Keywords: Power graph, Main eigenvalue, Cyclic group, Prime divisor.

Received: 15 April 2017, Accepted: 23 December 2017.

*Corresponding author: fathtabar@kashanu.ac.ir

(C) 2017 Yazd University. 
have a long history in the literature. One of the new consructions of graphs from a finite group $G$ is the power graph of $G$ which is the main concept considered in this paper. The undirected power graph $P(G)$ of $G$ is a graph with $V(P(G))=G$ and two distinct vertices $g_{1}$ and $g_{2}$ are adjacent if and only if there is a positive integer $k$ such that $g_{1}^{k}=g_{2}$ or $g_{1}=g_{2}^{k}$. The concept of the undirected power graph of the semigroups was first introduced by Chakrabarty et al. in 2009 [2]. They found that for any finite group $\mathrm{G}$, the power graph $P\left({ }_{n}\right)$ is always a connected graph. Then they presented a necessary and sufficient condition on the power graph to be a complete graph. Good results were later obtained in this area. For example, Cameron et al. observed that non-isomorphic finite groups may have isomorphic power graphs, but in the case of abelian finite groups they must be isomorphic [I]. Furthermore, Pourgholi, YousefiAzari and Ashrafi generalized some results of this area and gave some counterexamples on the conjecture of Chakrabarty which state that $P(G)$ is a Hamiltonian graph for all values of $n$ greater than 3 except for $n=2^{m} p_{1} p_{2} \ldots p_{k}$, where $p_{1}, p_{2}, \ldots, p_{k}$ are prime distinct divisors of $n$, $m$ and $k$ are no negative integers and $m \geq 2$ for $k=0,1$ and $k \geq 2$ for $m=0,1$ ([7]).

In this paper we deal with specific eigenvalues of the power graph of $\mathbb{Z}_{n}$ called main eigenvalues. An eigenvalue $\lambda$ of $(0,1)$-adjacency matrix $A$ of a finite graph $\Gamma$ with $n$ vertices is a main eigenvalue if it has a corresponding eigenvector $X$ which is not orthogonal to all-one vector $\mathbf{j}$. By Perron Frobenuis theorem, the simple largest eigenvalue of $\Gamma$ is always main, because the corresponding eigenvector can be chosen to have all positive components. For more studies on

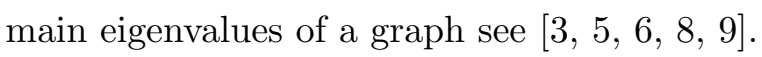

\section{Preliminaries}

Let $A$ have the following spectral decomposition

$$
A=\lambda_{1} P_{1}+\lambda_{2} P_{2}+:::+\lambda_{k} P_{k},
$$

where $\lambda_{1}, \lambda_{2}, \ldots, \lambda_{k}$ are the main eigenvalues of $\Gamma$ and $P_{1}, P_{2}, \ldots, P_{k}$ are the orthogonal projections onto eigenspaces $\varepsilon\left(\lambda_{1}\right), \varepsilon\left(\lambda_{2}\right), \ldots, \varepsilon\left(\lambda_{k}\right)$, respectively. Let $\Gamma$ be a simple graph with $(0,1)$-adjacency matrix $A$ and $W(\Gamma)=\left(w_{i j}\right)$ is its walk matrix where $w_{i j}$ is the number of walks of length $j$ with the start point $v_{i}$ and $\mathbf{j}$ be the all-one vector. We can easily see that:

$$
W(\Gamma)=\left[\begin{array}{llllllll}
\mathbf{j} & A \mathbf{j} & A^{2} \mathbf{j} & A^{3} \mathbf{j} & \ldots & A^{k} \mathbf{j} & \ldots & A^{n-1} \mathbf{j}
\end{array}\right] .
$$

We have the following theorem by [5] .

Theorem 2.1. The rank of $W(\Gamma)$ is equal to the number of main eigenvalues of $\Gamma$.

The number of main eigenvalues of $A$ can explain some of the graph theoretic properties of $\Gamma$. The following theorems give us some basic information about the structure of $\Gamma$ according to the number of its main eigenvalues: 
Theorem 2.2. [9] Let $\Gamma$ be a simple graph, $\Gamma$ has exactly one main eigenvalue if and only if $\Gamma$ is a regular graph. Particularly if $\Gamma$ is a $k$-regular graph, then $k$ is a simple eigenvalue of $\Gamma$.

Theorem 2.3. [5] Let $u$ and $v$ be two distinct vertices of $\Gamma$ such that $d(u) \neq d(v), N(u)$ and $N(v)$ are respectively the sets of neighbors of $u$ and $v$ and

$$
a=\frac{s(u)-s(v)}{d(u)-d(v)}, b=\frac{d(v) s(u)-d(u) s(v)}{d(u)-d(v)},
$$

where $s(u)=\sum_{w \in N(u)} d(w)$ and $s(v)=\sum_{w^{\prime} \in N(v)} d\left(w^{\prime}\right)$. Then $\Gamma$ has exactly two main eigenvalues $\lambda_{1,2}$ if and only if $A^{2} \mathbf{j}-a A \mathbf{j}+b \mathbf{j}=0$ and these two main eigenvalues are

$$
\lambda_{1,2}=\frac{a \pm \sqrt{a^{2}-4 b}}{2} .
$$

Theorem 2.4. [8] If $\Gamma$ has s main eigenvalues, then the automorphism group of $\Gamma$ has at least $s$ orbits in $V(\Gamma)$.

Now, let $P\left(\mathbb{Z}_{n}\right)$ be the power graph of the cyclic group of integers modulo $n$ under usual addition and $n=p_{1}^{r_{1}} p_{2}^{r_{2}} \ldots p_{s}^{r_{s}}$, where $p_{1}, p_{2}, \ldots, p_{s}$ are not necessarily pairwise distinct prime divisors of $n$ and $r_{1}, r_{2}, \ldots, r_{s}$ are positive integers. In the following section we find a condition on $n$ such that $P\left(\mathbb{Z}_{n}\right)$ have exactly one main eigenvalues. In section 3 , the number of main eigenvalues of $P\left(\mathbb{Z}_{n}\right)$ When all the above $p_{i}$ for $i=1,2, \ldots s$ are distinct are calculated.

\section{THE POWER GRAPH OF $\mathbb{Z}_{n}$}

As we mentioned before, if $G$ is a cyclic group generated by $a \in G$, then $\Gamma=P(G)$ is an undirected graph with vertex set $V(\Gamma)=G$ and two different vertices $u$ and $v$ are adjacent if and only if $\langle u\rangle \subseteq\langle v\rangle$ or $\langle v\rangle \subseteq\langle u\rangle$. Obviously, if $G$ is a finite group, then $P(G)$ is a connected graph. In [2], it was shown the power graph of a semigroup $S$ is complete if and only if for any two subsemigroups $S_{1}$ and $S_{2}$ of $S$ we have $S_{1} \subseteq S_{2}$ or $S_{2} \subseteq S_{1}$. So we have the following theorem:

Theorem 3.1. For every finite group $G, P(G)$ is a complete graph if and only if $G$ is cyclic group of order 1 or $p^{m}$, where $p$ is a prime number and $m \in \mathbb{N}$.

We know that the elements of the group of units in the integers modulo $n$ (i.e. $n$ ) are the generators of $\mathbb{Z}_{n}$. So the elements of ${ }_{n} \bigcup\{0\}$ are adjacent to all other vertices of $P\left(\mathbb{Z}_{n}\right)$. The number of these vertices are

$$
{ }_{n} \bigcup\{0\} \mid=\varphi(n)+1 \text {. }
$$

On the other hand, for every $d \mid n, d_{n}=\left\{d x ; x \in U_{n}\right\}$ is a clique of $P\left(\mathbb{Z}_{n}\right)$. If $x, y$ are two arbitrary distinct elements of ${ }_{n}$, then there is a unique element $r$ such that $r x=y$. So $d r x=d y$ and then $d x$ and $d y$ are adjacent in $P(G)$, which means $d_{n}$ is a clique of $P(G)$. 
Also, for every two cliques $d_{1 n}$ and $d_{2 n}\left(d_{1} \neq d_{2}\right)$, if one of the vertices of $d_{1 n}$ is adjacent to $d_{2} y \in d_{2 n}$, then all of the vertices of $d_{1 n}$ are adjacent to $d_{2} y$. Let $d_{1} x \in d_{1 n}$ be adjacent to $d_{2} y \in d_{2 n}$. Then $d_{1} x=m_{2} y$. So, for every $d_{1} z \in d_{1 n}$ we have,

$$
d_{1} z=d_{1} r x=r d_{1} x=r m d_{2} y=m^{\prime} d_{2} y
$$

Thus $d_{1} z$ is adjacent to $d_{2} y$, and we can easily see that,

$$
\left|d_{n}\right|=\left|U_{\left(\frac{n}{d}\right)}\right|=\varphi\left(\frac{n}{d}\right)
$$

Example 2.1. In the Figure 1 we draw the power graph of $\mathbb{Z}_{12}$ in order to see the overall picture of the power graph. As you can see in this case $n=2^{2} .3$ and graph has 5 cliques ${ }_{12} \bigcup\{0\}, 2_{12}, 3_{12}, 2_{12}^{2}$ and $2.3_{12}$. Note that the edges between circles connect all the vertices

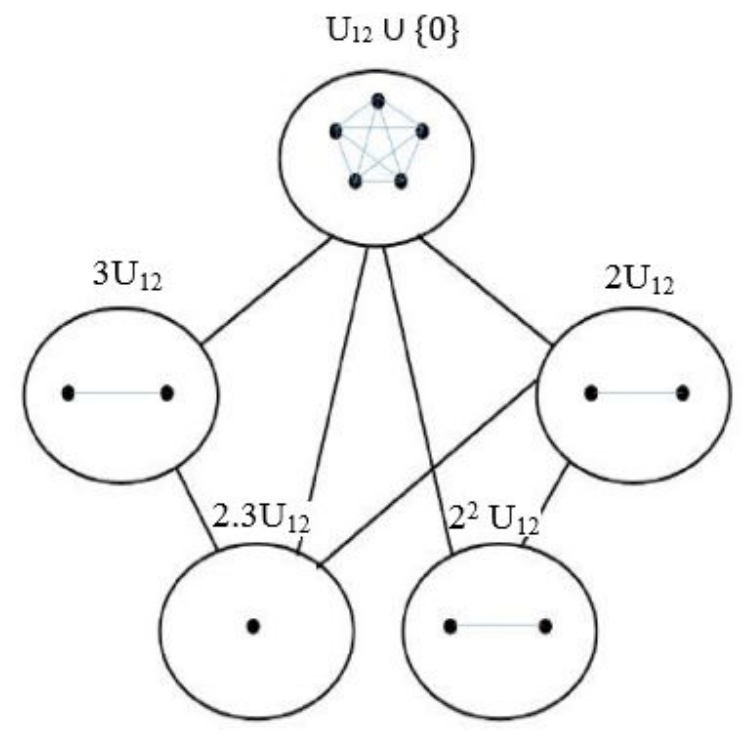

FiguRE 1. The power graph of $\mathbb{Z}_{12}$.

of one clique to all vertices of other clique.

Definition 3.2. An equitable partition of a graph $\Gamma$ is a partition of vertices into $C_{1}, C_{2}, \ldots, C_{s}$ parts such that for every $1 \leq i, j \leq s$, there exists a nonegative integer $b_{i j}$ such that every vertex $v_{i}$ in $C_{i}$, regardless of the choice of $v_{i}$, has exactly $b_{i j}$ neighbors in $C_{j}$. Therefore the induced subgraph of $\Gamma\left[C_{i}\right]$ is $b_{i i}$-regular.

Assume that $O_{1}, O_{2}, \ldots, O_{s}$ are the orbits of the automorphism group of $\Gamma$ in $V(\Gamma)$. This partition of vertices into orbits is called the orbit partition of $V(\Gamma)$. The following relationship is established between these two types of partitions.

Theorem 3.3. Every orbit partition is an equitable partition. 
Proof. The proof is simple. Consider two vertices $x, y$ in $O_{i}$. Then there is an automorphism $\varphi$ such that $\varphi(x)=y$. So $x$ and $y$ have the same number of neighbors in $O_{j}$ because $\varphi$ maps $O_{j}$ to $O_{j}$ preserving vertices degrees. Thus the orbit partition is an equitable partition.

As you can see in Figure W, every clique is an orbit. So according to the previous theorem, $\pi=\left\{{ }_{12} \bigcup\{0\}, 2_{12}, 2_{12}^{2}, 2.3_{12} ; 3_{12}\right\}$ is the equitable partition of $V(\Gamma)$. Note that in the general case, every equitable partition is not always an orbit partition. Consider the following graph, You can see $\{1,4\}$ and $\{2,3,5,6,7,8\}$ formed an equitable partition for the following graph
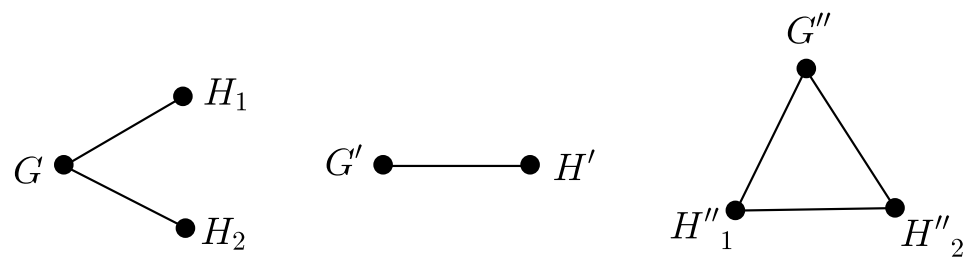

FIGURE 2. An example of equitable partition of vertices which is not an orbit partition.

which are not orbits. In the next section we prove new theorems about the main eigenvalues of $P\left(\mathbb{Z}_{n}\right)$ using the concept of the equitable partitions of vertices.

\section{MAIN THEOREMS}

Let's begin this section with an important theorem about $P\left(\mathbb{Z}_{n}\right)$ having exactly one main eigenvalue

Theorem 4.1. $P\left(\mathbb{Z}_{n}\right)$ has exactly one main eigenvalue if and only if $n=p^{m}$, where $p$ is a prime number and $m \in \mathbb{N}$.

Proof. If $P\left(\mathbb{Z}_{n}\right)$ has exactly one main eigenvalue, then $P\left(\mathbb{Z}_{n}\right)$ is a regular graph by [2.2. Clearly, a regular power graph is complete. So by $[],. n=p^{m}$, where $p$ is a prime number and $m$ is a nonegative integer.

Conversely, let $n=p^{m}$ where $p$ is a prime number and $m$ is a nonegative integer. So $P\left(\mathbb{Z}_{n}\right)$ is a complete graph, by $\mathbf{B}$. On the other side, $k$-regular graphs has exactly one main eigenvalue $k$, by [2.2. Since every complete graph with $n$ vertices is $n-1$-regular graph, $P\left(\mathbb{Z}_{n}\right)$ has exactly one main eigenvalue.

Definition 4.2. Let $\Gamma$ be simple graph and $\pi=V_{1}, V_{2}, \ldots, V_{k}$ is a partition of $V(\Gamma)$. Then a divisor of $\Gamma$ denoted by $\Gamma / \pi$ is a multidigraph with $k$ vertices correspond to $V_{1}, V_{2}, \ldots, V_{k}$ and $\left|N\left(v_{i}\right)\right|$ arcs from $V_{i}$ to $V_{j}$, where $\left|N\left(v_{i}\right)\right|$ is the number of neighbors of an arbitrary vertex $v_{i}$ of $V_{i}$ in $V_{j}$. 
Suppose that $\pi$ is an equitable partition of $V(\Gamma)$ according to the Definition 3.2. Every walk in $\Gamma / \pi$ has a homomorphic projection in $\Gamma$. But different walks in $\Gamma$ may have the same homomorphic projections in $\Gamma / \pi$. So by fixing a point in every part of $\pi$ as a start point, we have a one to one correspondence between the walks of $\Gamma$ and the walks of $\Gamma / \pi$. The following theorem demonstrates this fact.

Theorem 4.3. [3] The spectrum of every divior of $\Gamma$ includes all the main eigenvalues of $\Gamma$.

The definition of main eigenvalue of $\Gamma / \pi$ is quite similar to the definition of main eigenvalue of $\Gamma$.

Definition 4.4. [9] If $\pi=V_{1}, V_{2}, \ldots, V_{k}$ is an equitable partition of $\Gamma$ with $n$ vertices such that for every $1 \leq i \leq k,\left|V_{i}\right|=n_{i}$, then an eigenvalue $\lambda$ of $\Gamma / \pi$ is a main eigenvalue of $\Gamma / \pi$ if (1) $\lambda$ has a right eigenvector which is not orthogonal to the vector $\mathbf{j}(n)$, where

$$
\mathbf{j}(n)=\left(n_{1} \ldots n_{k}\right) .
$$

(2) $\lambda$ has a left eigenvector which is not orthogonal to the vector $\mathbf{j}$.

If $\pi$ is the discrete partition, the above definition is equivalent to that of main eigenvalue of a graph.

The following result is due to Teranishi [9].

Theorem 4.5. Let $\Gamma$ be a simple graph and $\pi$ is an equitable partition of the vertices of $\Gamma$. Then an eigenvalue $\lambda$ is a main eigenvalue of $\Gamma$ if and only if $\lambda$ is a main eigenvalue of $\Gamma / \pi$.

If $n=p_{1}^{r} p_{2}$, then by the above theorem we can calculate the number of main eigenvalues of $P\left(\mathbb{Z}_{n}\right)$ by finding the number of main eigenvalues of $P\left(\mathbb{Z}_{n}\right) / \pi$. Note that when using the above theorem, $\pi$ should be the equitable partition consisting of the elements of the set $\left\{d_{i n} ; d_{i} \mid n\right\}$. 
The walk matrix of figure 1 with $\mathbf{j}, A\left(P\left(\mathbb{Z}_{12}\right)\right) \mathbf{j}, \ldots$ and $A^{11}\left(P\left(\mathbb{Z}_{12}\right)\right) \mathbf{j}$ as its columns is as follows by use of Matlab 2015b.

$$
W\left(P\left(\mathbb{Z}_{12}\right)\right)=\left[\begin{array}{cccccccc}
1 & 11 & 101 & 973 & 9257 & 88363 & \cdots & 1.0 e+010 * 6.6562 \\
1 & 11 & 101 & 973 & 9257 & 88363 & \cdots & 1.0 e+010 * 6.6562 \\
1 & 11 & 101 & 973 & 9257 & 88363 & \cdots & 1.0 e+010 * 6.6562 \\
1 & 11 & 101 & 973 & 9257 & 88363 & \cdots & 1.0 e+010 * 6.6562 \\
1 & 11 & 101 & 973 & 9257 & 88363 & \cdots & 1.0 e+010 * 6.6562 \\
1 & 7 & 71 & 663 & 6355 & 60515 & \cdots & 1.0 e+010 * 4.5603 \\
1 & 7 & 71 & 663 & 6355 & 60515 & \cdots & 1.0 e+010 * 4.5603 \\
1 & 9 & 89 & 843 & 8061 & 76853 & \cdots & 1.0 e+010 * 5.791 \\
1 & 9 & 89 & 843 & 8061 & 76853 & \cdots & 1.0 e+010 * 5.791 \\
1 & 9 & 87 & 825 & 7877 & 75113 & \cdots & 1.0 e+010 * 5.6592 \\
1 & 8 & 81 & 764 & 7315 & 69722 & \cdots & 1.0 e+010 * 5.2539 \\
1 & 8 & 81 & 764 & 7315 & 69722 & \cdots & 1.0 e+010 * 5.2539
\end{array}\right] .
$$

The walk matrix of a qoutient graph $P\left(\mathbb{Z}_{12}\right) / \pi$ where $\pi$ is an equitable partition shown in figure 1 is

$$
W\left(P\left(\mathbb{Z}_{12}\right) / \pi\right)=\left[\begin{array}{cccccccc}
1 & 11 & 101 & 973 & 9257 & 88363 & \cdots & 1.0 e+010 * 6.6562 \\
1 & 7 & 71 & 663 & 6355 & 60515 & \cdots & 1.0 e+010 * 4.5603 \\
1 & 9 & 89 & 843 & 8061 & 76853 & \cdots & 1.0 e+010 * 5.791 \\
1 & 9 & 87 & 825 & 7877 & 75113 & \cdots & 1.0 e+010 * 5.6592 \\
1 & 8 & 81 & 764 & 7315 & 69722 & \cdots & 1.0 e+010 * 5.2539
\end{array}\right]
$$

As we can see, the rows of $W\left(P\left(\mathbb{Z}_{12}\right) / \pi\right)$ are the same as $W\left(P\left(\mathbb{Z}_{12}\right)\right)$ without repeated ones. Clearly

$$
\operatorname{rank}\left(W\left(P\left(\mathbb{Z}_{12}\right)\right)\right)=\operatorname{rank}\left(W\left(P\left(\mathbb{Z}_{12}\right) / \pi\right)\right)
$$

Let $n_{i}=\left|d_{i n}\right|=\varphi\left(\frac{n}{d_{i}}\right)$, where

$$
d_{i}=\left\{\begin{array}{ll}
p_{1}^{i-1} & i=1,2, \ldots, r+1 \\
p_{1}^{i-r-1} p_{2} & i=r+2, \ldots, 2 r
\end{array} .\right.
$$


Then,

$$
n_{i}=\left\{\begin{array}{lr}
p_{1}^{r-1}\left(p_{1}-1\right)\left(p_{2}-1\right)+1 & i=1 \\
p_{1}^{i-r}\left(p_{1}-1\right)\left(p_{2}-1\right) & i=2, \ldots, r \\
p_{2}-1 & i=r+1 \\
p_{1}^{i-4}\left(p_{1}-1\right) & i=r+2, \ldots, 2 r
\end{array} .\right.
$$

The adjacency matrix $A=\left[a_{i j}\right]$ of $P\left(\mathbb{Z}_{n}\right) / \pi=\left[a_{i j}\right]$ is defined as follows:

$$
a_{i j}=\left\{\begin{array}{lrl}
n_{j} & i \neq j, g c d\left(d_{i}, d_{j}\right) & \neq 1 \\
0 & i \neq j, g c d\left(d_{i}, d_{j}\right) & =1 \\
& i & =j
\end{array} .\right.
$$

Now, we are ready to prove the following theorem:

Theorem 4.6. $P\left(\mathbb{Z}_{p_{1} p_{2}}\right)$ has exactly three main eigenvalues.

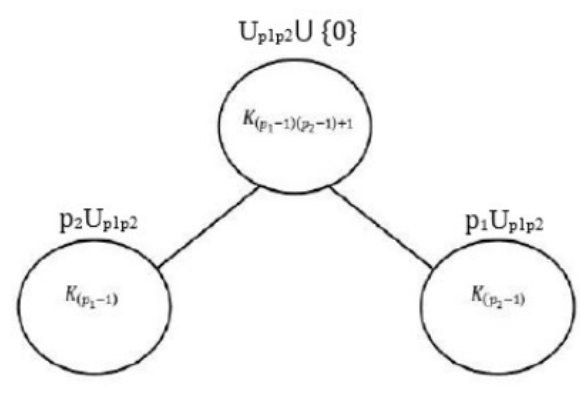

FiguRE 3. The power graph of $\mathbb{Z}_{p_{1} p_{2}}$

Proof. By looking at Figure [1, we can easily see that the automorphism group of $P\left(\mathbb{Z}_{p_{1} p_{2}}\right)$ has exactly three orbits $p_{1} p_{2} \bigcup\{0\}, p_{1 p_{1}-1 p_{2}}$ and $p_{2 p_{1} p_{2}}$. In this case the adjacency matrix of $P\left(\mathbb{Z}_{p_{1} p_{2}}\right) / \pi$ is:

$$
\mathrm{AG}_{\mathrm{E}}=\left[\begin{array}{ccc}
n_{1}-1 & n_{2} & n_{3} \\
n_{1} & n_{2}-1 & 0 \\
n_{1} & 0 & n_{3}-1
\end{array}\right]
$$

Let $\lambda$ be an eigenvalue of $A$ with corresponding eigenvector $X=\left[\begin{array}{lll}x_{1} & x_{2} & x_{3}\end{array}\right]^{t}$. We claim that $\lambda$ is a main eigenvalue of $P\left(\mathbb{Z}_{p_{1} p_{2}}\right)$. On the contrary, let $X$ is orthogonal to the vector $\mathbf{j}(n)$. So $n_{1} x_{1}+n_{2} x_{2}+n_{3} x_{3}=0$. Thus by $\mathrm{AG}_{\mathrm{E}} X=\lambda X$,

$$
\begin{aligned}
\left(n_{1}-1-\lambda\right) x_{1}+n_{2} x_{2}+n_{3} x_{3} & =0 \\
n_{1} x_{1}+\left(n_{2}-1-\lambda\right) x_{2} & =0 \\
n_{1} x_{1}+\left(n_{3}-1-\lambda\right) x_{3} & =0
\end{aligned}
$$


By solving the above equations system, we have $x_{1}=x_{2}=x_{3}=0$ which is a contradiction. Therefore, every eigenvalue of $A$ is main and $P\left(\mathbb{Z}_{p_{1} p_{2}}\right)$ has exactly three main eigenvalues.

There is another proof for Theorem 4.6] using Theorem [2.4. According to this theorem, A has at most three main eigenvalues. Since $P\left(\mathbb{Z}_{p_{1} p_{2}}\right)$ is not a regular graph, $P\left(\mathbb{Z}_{p_{1} p_{2}}\right)$ has more than one main eigenvalue by Theorem [2.2. But there is no power graph with exactly two main eigenvalues, because if there is a graph $\Gamma$ with exactly two main eigenvalues, we can choose two vertices $v$ and $u$ from two different classes of our desire equitable partition and see that $a$ and $b$ in Theorem $\left[2.3\right.$ are not unique which is a contradiction, too. So $P\left(\mathbb{Z}_{p_{1} p_{2}}\right)$ has exactly three main eigenvalues.

Now, let $W$ be a walk matrix of $P\left(\mathbb{Z}_{n}\right) / \pi$ with columns $\mathbf{j}, A \mathbf{j}, A^{2} \mathbf{j}, \ldots, A^{2 r} \mathbf{j}$. We rename the entries of these columns as follows

$$
A \mathbf{j}=\left[\begin{array}{c}
b_{1,1} \\
b_{1,2} \\
b_{1,3} \\
\vdots \\
b_{1,2 r+1}
\end{array}\right], A^{2} \mathbf{j}=\left[\begin{array}{c}
b_{2,1} \\
b_{2,2} \\
b_{2,3} \\
\vdots \\
b_{2,2 r+1}
\end{array}\right], \ldots, A^{2 r} \mathbf{j}=\left[\begin{array}{c}
b_{2 r, 1} \\
b_{2 r, 2} \\
b_{2 r, 3} \\
\vdots \\
b_{2 r, 2 r+1}
\end{array}\right] .
$$

The following relations hold for the entries of the vector $A \mathbf{j}$.

$$
b_{1, j}=\left\{\begin{array}{lr}
\left(\sum_{i=1}^{2 r+1} n_{i}\right)-1 & j=1 \\
\left(\sum_{i=1}^{2 r} n_{i}\right)-1 & j=2 \\
\left(\sum_{i=1, i \neq\{r+2, r+3, \ldots, r+j-1\}}^{2 r} n_{i}\right)-1, & 3 \leq j \leq r+1 \\
\left(\sum_{i=1, i \neq\{j-r+1, j-r+2, \ldots, r+1\}}^{2 r+1} n_{i}\right)-1, & r+2 \leq j \leq 2 r \\
\left(\sum_{i=1, i \neq\{2,3, \ldots, r+1\}}^{2 r+1} n_{i}\right)-1 & j=2 r+1
\end{array} .\right.
$$

Also there are some relations between the entries of $i$-th column and the entries of $i-1$-th column as follows

$$
b_{i, j}=\left\{\begin{array}{lr}
\left(n_{1}-1\right) b_{i-1,1}+\sum_{k=1, k \neq 1}^{2 r+1} n_{k} b_{i-1, k} & j=1 \\
\left(n_{2}-1\right) b_{i-1,2}+\sum_{k=1, k \neq 2}^{2 r} n_{k} b_{i-1, k} & j=2 \\
\left(n_{j}-1\right) b_{i-1, j}+\sum_{k=1, k \neq j,\{r+2, r+3, \ldots, r+j-1\}}^{2 r} n_{k} b_{i-1, k}, & 3 \leq j \leq r+1 \\
\left(n_{2} r+1-1\right) b_{i-1,2 r+1}+\sum_{k=1, k \neq 2 r+1,\{2,3, \ldots, r+1\}}^{2 r+1} n_{k} b_{i-1, k} & j=2 r+1
\end{array} .\right.
$$

By using Theorem $\llbracket$ we can see that,

$$
\operatorname{det}(W)=(-1)^{r} \prod_{i=2}^{2 r+1} n_{i} \operatorname{det}\left(V_{1}^{\prime}\right),
$$


where

$$
V_{1}^{\prime}=\left[\begin{array}{ccccc}
1 & 1-b_{1,2 r+1} & 1-b_{1,2 r+1}+b_{2,2 r+1} & \ldots & 1-b_{1,2 r+1}+b_{2,2 r+1}+\ldots+b_{2 r, 2 r+1} \\
1 & 1-b_{1, r+2} & 1-b_{1, r+2}+b_{2, r+2} & \ldots & 1-b_{1, r+2}+b_{2, r+2}+\ldots+b_{2 r, r+2} \\
1 & 1-b_{1, r+3} & 1-b_{1, r+3}+b_{2, r+3} & \ldots & 1-b_{1, r+3}+b_{2, r+3}+\ldots+b_{2 r, r+3} \\
\vdots & & & & \\
1 & 1-b_{1, r+r} & 1-b_{1, r+r}+b_{2, r+r} & \ldots & 1-b_{1, r+r}+b_{2, r+r}+\ldots+b_{2 r, r+r} \\
1 & 1-b_{1,3} & 1-b_{1,3}+b_{2,3} & \ldots & 1-b_{1,3}+b_{2,3}+\ldots+b_{2 r, 3} \\
1 & 1-b_{1,4} & 1-b_{1,4}+b_{2,4} & \ldots & 1-b_{1,4}+b_{2,4}+\ldots+b_{2 r, 4} \\
\vdots & & & & \\
1 & 1-b_{1, r+1} & 1-b_{1, r+1}+b_{2, r+1} & \ldots & 1-b_{1, r+1}+b_{2, r+1}+\ldots+b_{2 r, r+1} \\
1 & 1-b_{1,2} & 1-b_{1,2}+b_{2,2} & \ldots & 1-b_{1,2}+b_{2,2}+\ldots+b_{2 r, 2}
\end{array}\right]
$$

by Gaussian elimination and some factorizations. After $r$ times doing the same on $V_{1}^{\prime}$ we have,

$$
\operatorname{det}(W)=\prod_{i_{1}=2}^{2 r+1} n_{i_{1}} \prod_{i_{2}=2, i_{2} \neq r+1}^{2 r} n_{i_{2}} \prod_{i_{3}=i_{3} \neq r+1}^{2 r-1} n_{i_{3}} \ldots \prod_{i_{r}=r-1}^{r+2} n_{i_{r}} \operatorname{det}\left(V_{r}\right),
$$

If $r \neq 2$ is an even number, then the columns of $r \times r$ matrix $V_{r}$ are

$$
V_{1}=\left[\begin{array}{c}
v_{11} \\
v_{12} \\
v_{13} \\
v_{14} \\
v_{15} \\
\vdots \\
v_{1 r}
\end{array}\right], V_{2}=\left[\begin{array}{c}
v_{21} \\
v_{22} \\
v_{23} \\
v_{24} \\
v_{25} \\
\vdots \\
v_{2 r}
\end{array}\right], V_{3}=\left[\begin{array}{c}
v_{31} \\
v_{32} \\
v_{33} \\
v_{34} \\
v_{35} \\
\vdots \\
v_{3 r}
\end{array}\right], \cdots, V_{r}=\left[\begin{array}{c}
v_{r 1} \\
v_{r 2} \\
v_{r 3} \\
v_{r 4} \\
v_{r 5} \\
\vdots \\
v_{r r}
\end{array}\right],
$$

where

and if $r=2$

$$
V_{r}=\left[\begin{array}{cc}
-n_{3}+n_{5} & n_{3}\left(1-b_{1,3}\right)-n_{5}\left(1-b_{1,5}\right) \\
n_{3} n_{4}-n_{2} n_{5} & -n_{3} n_{4}\left(1-b_{1,4}\right)+n_{2} n_{5}\left(1-b_{1,2}\right)
\end{array}\right] .
$$

Now, we have the following theorem:

Theorem 4.7. Let $n=p_{1}^{r} p_{2}$, where $p_{1}$ and $p_{2}$ are distinct prime divisors of $n$. Then the power graph $P\left(\mathbb{Z}_{n}\right)$ has exactly $2 r+1$ main eigenvalues. 


\begin{tabular}{|c|c|}
\hline$v_{11}$ & $-\sum_{3}^{r+1} n_{i}+\sum_{r+2}^{2 r} n_{i}+n_{2 r+1}$ \\
\hline$v_{12}$ & $n_{r+1} n_{2 r}-n_{2} n_{2 r+1}$ \\
\hline$v_{13}$ & $-n_{r+1} n_{2 r} n_{r}+n_{2} n_{2 r+1} n_{r+2}$ \\
\hline$\vdots$ & $\vdots$ \\
\hline$v_{1 r}$ & $n_{r+1} n_{2 r} n_{r} n_{2 r-1} n_{r-1} n_{2 r-2} n_{r-2} \ldots n_{(3 r / 2+1)}-n_{2} n_{2 r+1} n_{r+2} n_{3} n_{r+3} n_{4} \ldots n_{(r / 2+1)}$ \\
\hline$v_{21}$ & $\sum_{3}^{r+1} n_{i}\left(1-b_{1 i}\right)-\sum_{r+2}^{2 r} n_{i}\left(1-b_{1 i}\right)-n_{2 r+1}\left(1-b_{12 r+1}\right)$ \\
\hline$v_{22}$ & $-n_{r+1} n_{2 r}\left(1-b_{12 r}\right)+n_{2} n_{2 r+1}\left(1-b_{12}\right)$ \\
\hline$v_{23}$ & $n_{r+1} n_{2 r} n_{r}\left(1-b_{1 r}\right)-n_{2} n_{2 r+1} n_{r+2}\left(1-b_{1 r+2}\right)$ \\
\hline$\vdots$ & $\vdots$ \\
\hline$v_{2 r}$ & $-n_{r+1} n_{2 r} n_{r} n_{2 r-1} n_{r-1} n_{2 r-2} n_{r-2} \cdots n_{(3 r / 2+1)}\left(1-b_{1(3 r / 2+1)}\right)$ \\
& $+n_{2} n_{2 r+1} n_{r+2} n_{3} n_{r+3} n_{4} \cdots n_{(r / 2+1)}\left(1-b_{1(r / 2+1)}\right)$ \\
\hline$v_{31}$ & $-\sum_{3}^{r+1} n_{i}\left(1-b_{1 i}+b_{2 i}\right)+\sum_{r+2}^{2 r} n_{i}\left(1-b_{1 i}+b_{2 i}\right)+n_{2 r+1}\left(1-b_{1 i}+b_{22 r+1}\right)$ \\
\hline$v_{32}$ & $n_{r+1} n_{2 r}\left(1-b_{12 r}+b_{22 r}\right)-n_{2} n_{2 r+1}\left(1-b_{12}+b_{22}\right)$ \\
\hline$v_{33}$ & $-n_{r+1} n_{2 r} n_{r}\left(1-b_{1 r}+b_{2 r}\right)+n_{2} n_{2 r+1} n_{r+2}\left(1-b_{1 r+2}+b_{2 r+2}\right)$ \\
\hline$\vdots$ & $\vdots$ \\
\hline$v_{3 r}$ & $n_{r+1} n_{2 r} n_{r} n_{2 r-1} n_{r-1} n_{2 r-2} n_{r-2} \cdots n_{(3 r / 2+1)}\left(1-b_{1(3 r / 2+1)}+b_{2(3 r / 2+1)}\right)$ \\
\hline$\vdots$ & $-n_{2} n_{2 r+1} n_{r+2} n_{3} n_{r+3} n_{4} \ldots n_{(r / 2+1)}\left(1-b_{1(r / 2+1)}+b_{2(r / 2+1)}\right)$ \\
\hline$v_{r 1}$ & $\vdots$ \\
\hline$v_{r 2}$ & $\sum_{3}^{r+1} n_{i}\left(1-\cdots-b_{r-1 i}\right)-\sum_{r+2}^{2 r} n_{i}\left(1-\cdots-b_{r-1 i}\right)-n_{2 r+1}\left(1-\cdots-b_{r-12 r+1}\right)$ \\
\hline$v_{r 3}$ & $-n_{r+1} n_{2 r}\left(1-\cdots-b_{r-12 r}\right)+n_{2} n_{2 r+1}\left(1-\cdots-b_{r-1 r+2}\right)$ \\
\hline$\vdots$ & $n_{r+1} n_{2 r} n_{r}\left(1-\cdots-b_{r-12 r}\right)-n_{2} n_{2 r+1} n_{r+2}\left(1-\cdots-b_{r-1 r+2}\right)$ \\
\hline$v_{r r}$ & $n_{r+1} n_{2 r} n_{r} n_{2 r-1} n_{r-1} n_{2 r-2} n_{r-2} \cdots n_{(3 r / 2+1)}\left(1-\cdots-b_{r-1(3 r / 2+1)}\right)$ \\
\hline & $-n_{2 r+1} n_{r+2} n_{3} n_{r+3} n_{4} \cdots n_{(r / 2+1)}\left(1-\cdots-b_{r-1(r / 2+1)}\right)$ \\
\hline
\end{tabular}

FiguRE 4 . The values of $v_{p q}$ for $1 \leq p, q \leq r$

Proof. We begin the proof with the case of $r=2$. Using the Theorems 4.5 and, it is enough to show that $\operatorname{det}\left(V_{1}\right) \neq 0$. By simple calculations we can see that,

$$
\begin{aligned}
\operatorname{det}\left(V_{r}\right) & =n_{3}^{2} n_{4}\left(b_{1,3}-b_{1,4}\right)+n_{5}^{2} n_{2}\left(b_{1,5}-b_{1,2}\right) \\
& +n_{2} n_{3} n_{5}\left(b_{1,2}-b_{1,3}\right)+n_{3} n_{4} n_{5}\left(b_{1,5}-b_{1,4}\right) .
\end{aligned}
$$

On the other side, by $\mathbb{8}$

$$
\operatorname{det}\left(V_{r}\right)=n_{2}\left(n_{3}^{2}-n_{2}-n_{3} n_{5}+2 n_{2} n_{5}-n_{2} n_{5}^{2}-n_{3} n_{5}^{2}+n_{5}^{3}\right) .
$$


But $N=n_{3}^{2}-n_{2}-n_{3} n_{5}+2 n_{2} n_{5}-n_{2} n_{5}^{2}-n_{3} n_{5}^{2}+n_{5}^{3}>0$, since

$$
\begin{aligned}
N & =n_{3}^{2}-n_{2}-n_{3} n_{5}+2 n_{2} n_{5}-\left(n_{2}+n_{3}\right) n_{5}^{2}+n_{5}^{3} \\
& >n_{3}\left(n_{3}-n_{5}\right)+n_{2}\left(2 n_{5}-1\right)-n_{5}^{3}+n_{5}^{3} \\
& >n_{3}\left(n_{3}-n_{5}\right)+n_{3}\left(2 n_{5}-1\right) \\
& =n_{3}\left(n_{5}+n_{3}-1\right) \\
& >0 .
\end{aligned}
$$

Now, let $r \neq 2$. By considering the elements in the first top row and the respective minors $A_{0}, A_{1}, \ldots$ and $A_{r-1}$ we have

$$
\begin{aligned}
\operatorname{det}\left(V_{r}\right)= & +\left[-\sum_{3}^{r+1} n_{i}+\sum_{r+2}^{2 r-1} n_{i}+n_{2 r+1}\right] A_{0} \\
& -\left[-\sum_{3}^{r+1} n_{i} b_{1, i}+\sum_{r+2}^{2 r-1} n_{i} b_{1, i}+n_{2 r+1} b_{1,2 r+1}\right] A_{1} \\
& +\left[-\sum_{3}^{r+1} n_{i} b_{2, i}+\sum_{r+2}^{2 r-1} n_{i} b_{2, i}+n_{2 r+1} b_{2,2 r+1}\right] A_{2} \\
& -\ldots \\
& -\left[\sum_{3}^{r+1} n_{i} b_{r-1, i}+\sum_{r+2}^{2 r-1} b_{r-1, i}+n_{2 r+1} b_{r-1,2 r+1}\right] A_{r-1} .
\end{aligned}
$$

Assume that for every $i=3, \ldots, 2 r+1, b_{0, i}=1$. Then for every $0 \leq r \leq 1$, we have,

$$
-\sum_{3}^{r+1} n_{i} b_{j, i}+\sum_{r+2}^{2 r-1} n_{i} b_{j, i}+n_{2 r+1} b_{j, 2 r+1}>0 .
$$


On the other hand, for every permutation $\sigma=(\sigma(1), \sigma(2), \ldots, \sigma(r))$ in the symetric group $S_{r}$ and every $A_{s},(0 \leq s \leq r-1)$, the following holds

$$
\begin{aligned}
& A_{s}=\prod_{i=0}^{r / 2-2} \prod_{i_{1}=r-i}^{r+1} n_{i_{1}} \prod_{i_{2}=2 r-i}^{2 r} n_{i_{2}}^{r / 2-1} \prod_{j=0}^{r+1} \prod_{j_{1}=r-j+1}^{r+1} n_{j_{1}} \prod_{j_{2}=2 r-j}^{2 r} n_{j_{2}} \\
& \sum_{\sigma \in S_{r}, \sigma(1)=s+1}(-1)^{r+s} \prod_{k=1}^{r / 2} b_{\sigma(2 k)-1,2 r-(k-1)} \prod_{l=1}^{r / 2-1} b_{\sigma(2 l+1)-1, r-l} \\
& +\sum_{k_{1}=0}^{r / 2-1} \prod_{i=0}^{r / 2-2} \prod_{i_{1}=r-i}^{r+1} n_{i_{1}} \prod_{i_{2}=2 r-i}^{2 r} n_{i_{2}} \prod_{j=0, j \neq k_{1}}^{r / 2-1} \prod_{j_{1}=r-j+1}^{r+1} n_{j_{1}} \prod_{j_{2}=2 r-j}^{2 r} n_{j_{2}} \\
& \sum_{\sigma \in S_{r}, \sigma(1)=s+1}(-1)^{r+s} \prod_{k=1, k \neq k_{1}+1}^{r / 2} b_{\sigma(2 k)-1,2 r-(k-1)} \prod_{l=1}^{r / 2-1} b_{\sigma(2 l+1)-1, r-l} b_{\sigma\left(2\left(k_{1}+1\right)-1, k_{1}+2\right.} \\
& +\sum_{k_{1}=0}^{r / 2-2} \prod_{i=0, i \neq k_{1}}^{r / 2-2} \prod_{i_{1}=r-i}^{r+1} n_{i_{1}} \prod_{i_{2}=2 r-i}^{2 r} n_{i_{2}} \prod_{j=0}^{r / 2-1} \prod_{j_{1}=r-j+1}^{r+1} n_{j_{1}} \prod_{j_{2}=2 r-j}^{2 r} n_{j_{2}} \prod_{\alpha_{1}=2}^{k_{1}+2} n_{\alpha_{1}} \prod_{\beta_{1}=r+2}^{k_{1}+r+2} n_{\beta_{1}} n_{2 r+1} \\
& \sum_{\sigma \in S_{r}, \sigma(1)=s+1}(-1)^{r+s} \prod_{k=1}^{r / 2} b_{\sigma(2 k)-1,2 r-(k-1)} \prod_{l=1, l \neq k_{1}+1}^{r / 2-1} b_{\sigma(2 l+1)-1, r-l} b_{\sigma\left(2\left(k_{1}+1\right)-1, k_{1}+2\right.} \\
& +\ldots \\
& +\prod_{i=0}^{r / 2-2} \prod_{\alpha_{1}=2}^{i+2} n_{\alpha_{1}} \prod_{\beta_{1}=r+2}^{i+r+2} n_{1}\left(n_{2 r+1}\right)^{r / 2-1} \prod_{j=0}^{r / 2-1} \prod_{\alpha_{2}=2}^{j+2} n_{\alpha_{2}} \prod_{\beta_{2}=r+2}^{j+r+2} n_{2}\left(n_{2 r+1}\right)^{r / 2} \\
& \sum_{\sigma \in S_{r}, \sigma(1)=s+1}(-1)^{r+s} \prod_{k=1}^{r / 2} b_{\sigma(2 k)-1, k+1} \prod_{l=1}^{r / 2-1} b_{\sigma(2 l+1)-1, r+l+1)} .
\end{aligned}
$$

By using of (国), (可) and (四) in the above relations, we can see that for every even number $s$, $A_{s}$ has a positive value and for every odd number $s, A_{s}$ has a negative value. This shows that we always have $\operatorname{det}\left(V_{r}\right)>0$. By the same way we can show that if $r$ is an odd number then $\operatorname{det}\left(V_{r}\right)>0$. So the proof is complete and $P\left(\mathbb{Z}_{p_{1}^{r} p_{2}}\right)$ has exactly $2 r+1$ main eigenvalues.

Conjecture. Every power graph $P\left(\mathbb{Z}_{n}\right)$ has exactly

$$
r=\sum_{i=1}^{k} r_{i}+\prod_{i=1}^{k} r_{i}+\sum_{i_{1}, i_{2}=1, i_{1}<i_{2}}^{k} r_{i_{1}} r_{i_{2}}+\ldots+\sum_{i_{1}, \ldots, i_{k}=1, i_{1}<\ldots<i_{k}}^{k} r_{i_{1}} \ldots r_{i_{k}}
$$

main eigenvalues, where $n=p_{1}^{r_{1}} p_{2}^{r_{2}} p_{3}^{r_{3}} \ldots p_{k}^{r_{k}}$.

\section{ACKNOWLEDGments}

The research of the second author was supported by the University of Kashan (Grant No.159021/16). 


\section{REFERENCES}

[1] P.J. Cameron and Sh. Ghosh, The power graph of a finite group, Discrete Math., Vol. 311 Issue 13 (2011), pp. $1220-1222$.

[2] I. Chakrabarty, Sh. Ghosh and M. K. Sen, Undirected power graphs of semigroups, Semigroup Forum, Vol. 78 Issue 3 (2009), pp. 410-426.

[3] D. Cvetković, The main part of the spectrum, divisors and switching of graphs, Publ. Inst. Math. (Beograd) (N.S.), Vol. 37 (1978), pp. 31-38.

[4] D. Cvetković, P. Rowlinson and S. Simić, Eigenspaces of graphs, Encyclopedia of Mathematics and its Applications, 66. Cambridge University Press, Cambridge, (1997).

[5] E. Hagos, Some results on graph spectra, Linear Algebra Appl., Vol 356 (2002), pp. 103-111.

[6] V. Nikiforov, Walks and the spectral radius of graphs, Linear Algebra Appl., Vol. 418 Issue 1 (2006), pp. 257-268.

[7] G. R. Pourgholi, H. Yousefi-Azari and A. R. Ashrafi, The undirected power graph of a finite group, Bull. Malays. Math. Sci. Soc., Vol.38 Issue 4 (2015), pp. 1517-1525.

[8] P. Rowlinson, The main eigenvalues of a graph: a survey, Appl. Anal. Discrete Math., Vol. 1 No. 2 (2007), pp. $445-471$.

[9] Y. Teranishi, Main eigenvalues of a graph, Linear and Multilinear Algebra, Vol. 49 Issue 4 (2001), pp. 289-303.

\section{Mehrnoosh Javarsineh}

Department of Pure Mathematics, Faculty of Mathematical Sciences, University of Kashan,

Kashan 87317-53153, Iran.

mehrnooshjavar@gmail.com

\section{Gholam Hossein Fath-Tabar}

Department of Pure Mathematics, Faculty of Mathematical Sciences, University of Kashan, Kashan 87317-53153, Iran.

fathtabar@kashanu.ac.ir 